\title{
EXAMEN PSICOMÉTRICO EXPLORATORIO DEL MILLON CLINICAL MULTIAXIAL INVENTORY III (MCMI-III) EN POBLACIÓN PENITENCIARIA CHILENA
}

EXPLORATORY PSYCHOMETRIC EXAMINATION OF THE MILLON CLINICAL MULTIAXIAL INVENTORY III (MCMI-III) IN CHILEAN PRISON INMATES

DOI: 10.22199/S07187475.2013.0002.00005

Recibido: 20 de Mayo del 2013 | Aceptado: 10 de Julio del 2013

\author{
MAURICIO SOUCI V.1; EUGENIA V. VINET2 \\ (UNIVERSIDAD DE LA FRONTERA, Temuco, Chile)
}

\begin{abstract}
RESUMEN
Este estudio exploró las propiedades psicométricas del MCMI-III en una muestra $(\mathrm{n}=123)$ de ofensores masculinos adultos chilenos recluidos por la comisión de delitos sexuales, delitos contra las personas, y delitos contra la propiedad. Específicamente, examinó su validez concurrente y capacidad discriminante, así como su confiabilidad. Las escalas de personalidad del MCMI-III tuvieron moderada validez concurrente con las dimensiones del Modelo de Cinco Factores medidas por el NEO-FFI; además, el MCMI-III demostró adecuada capacidad discriminante cuando los sujetos recluidos fueron comparados con pacientes clínicos. Sin embargo, el MCMI-III mostró modesta consistencia interna y estabilidad temporal, lo cual advirtió que la motivación, oposicionismo y deseabilidad social son variables relevantes en ofensores recluidos. Estos hallazgos, parcialmente consistentes con los reportados por otros investigadores, sugieren que el MCMI-III puede ser un instrumento útil para evaluar el funcionamiento psicológico de sujetos forenses chilenos pero que se requiere trabajo adicional para evaluar sus propiedades psicométricas en esta población.
\end{abstract}

PALABRAS CLAVE: MCMI-III; confiabilidad; validez; reclusos chilenos

\section{ABSTRACT}

This study explores the psychometric properties of the MCMI-III in a sample $(n=123)$ of Chilean adult male offenders imprisoned for the perpetration of property, personal, and sexual offenses. Specifically, it examined the MCMI-III's concurrent and discriminant validity as well as its reliability. The MCMI-III personality scales had moderate concurrent validity with the dimensions of the Five-Factor Model as measured by the NEO-FFI, but they had an adequate discriminant capacity when the prison inmates were compared with clinical patients. The MCMI-III also showed modest internal consistency and test-retest stability, which demonstrate that motivation, oppositional behavior and social desirability are relevant variables in prison inmates. These findings, which are partially consistent with those reported by other researchers, suggest that the MCMI-III may be a useful instrument to assess the psychological functioning of Chilean forensic populations, but further work is required to evaluate the test's psychometric properties in these populations.

KEY WORDS: MCMI-III; reliability; validity; Chilean prison inmates 
Características distintivas de la evaluación psicológica forense indican que: (a) probablemente el evaluado distorsionará deliberadamente lo que reporta de sí mismo, (b) hay incentivos situacionales para el evaluado que pueden incidir en su respuesta, y (c) los métodos pueden ser desafiados; siendo en este contexto muy importante el uso de tests psicológicos que demuestren niveles satisfactorios de validez y confiabilidad (Heilbrun, Marczyk, DeMatteo y Mack-Allen, 2007). Además, en psicología forense, las técnicas de evaluación más prometedoras en proporcionar solidez al testimonio psicológico experto respecto de trastornos mentales, han sido claramente inventarios clínicos de personalidad de autoreporte (Dyer, 2008).

El Inventario Clínico Multiaxial de Millon III (MCMI-III) es un autoreporte de evaluación integral de la personalidad para adultos, que evalúa los trastornos de personalidad descritos en el DSM-IV-TR (American Psychiatric Association, 2000) junto a otros que figuraban en el DSM-III-R (American Psychiatric Association, 1987). Este test ha sido desarrollado en Estados Unidos según el modelo de personalidad y psicopatología de Theodore Millon (Millon y Davis, 1998). El MCMI-III es bastante utilizado por psicólogos forenses (Archer, Buffington-Vollum, Stredny, y Handel, 2006); además cuenta con una versión chilena y estudios iniciales en población clínica chilena (Vinet, Saiz, Gempp, Benavente, Lutz y Pezoa, 1999) que dan cuenta de adecuados niveles de confiabilidad y validez compatibles con los reportados originalmente (Millon, 1994); sin embargo no existen reportes de sus propiedades psicométricas en poblaciones forenses chilenas.

A nivel internacional se han realizado diversos estudios con el MCMI-III y su versión previa MCMI-II. Específicamente en el MCMI-II los sujetos forenses han obtenido puntajes mayores que los sujetos clínicos en prácticamente todas las escalas (Cohen et al., 2002; Winberg y Vilalta, 2009), y el MCMI-III ha discriminado entre sujetos forenses (de centros de orientación forense y prisiones) y sujetos clínicos en sus diferentes escalas (Rossi, Van der Ark, y Sloore, 2007); sin embargo, en Chile no se cuenta aún con este tipo de estudios.

Atendiendo a estos antecedentes, este estudio examinó exploratoriamente las características psicométricas del MCMI-III en población penitenciaria chilena, particularmente su confiabilidad (consistencia interna y estabilidad temporal) y su validez de constructo utilizando como referente la validez concurrente con el Modelo de Cinco Factores (MCF) medido a través del cuestionario NEO-FFI (Costa y McCrae, 1989) y su capacidad de discriminación al comparar a los participantes con la muestra clínica chilena antes referida (Vinet et al., 1999).

Entre los antecedentes relevantes a considerar en este estudio están los siguientes:

\section{Evaluación en Poblaciones Forenses- Penitenciarias}

Hallazgos empíricos tanto extranjeros (Bennet, Holloway, y Farrington, 2008; Boden, Fergusson, y Horwood, 2012; Piquero, Farrington, y Blumstein, 2007) como nacionales (Cooper, 1994) reportan importantes variables sociodemográficas y judiciales asociadas a la comisión de delitos: edad (temprana) del inicio delictivo, nivel educacional (bajo) y fracaso escolar, estado civil predominantemente soltero, tipo de delito, número de delitos, consumo de alcohol y drogas.

Además, en el ámbito penitenciario cobra importancia la ansiedad que experimentan los internos como efecto del encarcelamiento, pues un buen conocimiento del medio rebaja la tensión emocional y un gran desconocimiento la eleva considerablemente (Bermúdez- 
Fernández, 2006). Otro elemento importante es la cercanía a la libertad, la cual permite operacionalizar el periodo de encarcelamiento en tres etapas: inicial, central, y final, las cuales varían en el grado de motivación, oposicionismo y conformidad con las normas sociales que presentan los sujetos privados de libertad (BermúdezFernández, 2006).

Con respecto al comportamiento frente a instrumentos psicológicos, se ha observado que cuando la evaluación parece irrelevante o absurda, los niveles de motivación en los sujetos disminuyen impactando adversamente en la validez de la evaluación (Kline, 2000). Además, hay que tener en cuenta algunos problemas de los inventarios de auto-reporte como deseabilidad social, respuestas extremas, respuestas al azar, motivación a la consistencia, que son vistos como específicos del contexto de evaluación (Paulhus y Vazire, 2007).

La personalidad es una variable compleja a examinar en poblaciones forenses. Este examen se realiza frecuentemente utilizando el constructo de trastornos de la personalidad, la nomenclatura del DSM y el MCMI en sus diversas versiones como instrumento de evaluación. Se ha observado, por ejemplo, que al operacionalizar los trastornos de personalidad según el MCMI-II, los hombres recluidos por violencia grave contra la mujer presentan mayor prevalencia del trastorno compulsivo, seguido del trastorno dependiente y del trastorno paranoide, mientras que el trastorno antisocial tiene baja frecuencia (Fernández-Montalvo y Echeburúa, 2008). En otros estudios, al evaluar la personalidad en sujetos denunciados por delitos de violencia de género, abuso sexual, violación u homicidio, se ha encontrado una alta prevalencia del trastorno compulsivo seguido de los trastornos narcisista, paranoide, dependiente, histriónico, antisocial, agresivo-sádico y limítrofe (Winberg y Vilalta, 2009). Para entender esta nomenclatura es necesario profundizar en el modelo de personalidad de Millon (Millon y Davis, 1998).

Modelo de Personalidad de Theodore Millon y MCMI-III

Millon concibe la personalidad como el patrón de funcionamiento adaptativo que los seres humanos presentan para relacionarse con su ambiente (Cardenal, Sánchez y Ortiz-Tallo, 2007). Basándose en la combinación de tres dimensiones polares (búsqueda de placer y evitación del dolor, modos de adaptación activo y pasivo, y orientación hacia sí mismo u otros), Millon postuló 11 patrones básicos de personalidad (esquizoide, evitador, agresivo-sádico, autodestructivo, dependiente, histriónico, narcisista, antisocial, compulsivo, pasivo-agresivo y depresivo), a ellos agregó tres patrones severos (esquizotípico, limítrofe $y$ paranoide), describiendo finalmente 14 patrones de personalidad (Millon y Davis, 1998).

En estos patrones, los rasgos de personalidad están distribuidos en un continuo de funcionamiento sano $y$ patológico, sin una línea divisoria, en el que se sitúan las variantes bien y mal ajustadas. Por un lado, la elevación de un elemento de una polaridad no implica un buen atributo, sino simplemente una tendencia hacia algún estilo de comportamiento; por otro lado, los patrones de personalidad normal y anormal comparten similares rasgos, siendo la principal diferencia que los últimos carecen de la habilidad de adaptación debido a rasgos rígidos (Millon y Davis, 1998; Strack y Millon, 2007).

Así como los patrones anormales están representados en el eje II del DSM-IV, Millon (1994) considera a los síndromes clínicos del eje I del DSM-IV como extensiones 0 distorsiones del patrón básico de personalidad, distinguiendo síndromes clínicos básicos y severos: los básicos son 
trastorno de ansiedad, histeriforme, hipomanía, neurosis depresiva, abuso de alcohol, abuso de drogas y trastorno de estrés postraumático, mientras que los severos son pensamiento psicótico, depresión mayor, y delirio psicótico.

Este modelo teórico posibilitó la construcción del MCMI-III (Millon, 1994). Este instrumento refleja, a través de sus 28 escalas, las agrupaciones ya descritas: las escalas de personalidad evalúan 11 patrones básicos y tres patrones graves de personalidad; las escalas de síndromes clínicos evalúan 11 alteraciones de tipo reactivo, generalmente transitorias y breves, que pueden ser moderadas o severas; estas escalas se complementan con tres escalas modificadoras utilizadas para controlar $y$ corregir tendencias de respuesta en las otras escalas y una escala de validez que puede detectar confusión mental, respuestas al azar, o negativismo extremo.

Las cualidades más distintivas del MCMI-III incluyen su compatibilidad con la nomenclatura del DSM-IV, sus adecuadas propiedades psicométricas, su corta longitud que permite minimizar la fatiga del sujeto, y la sencillez de sus ítems redactados para un nivel lector de octavo grado o menos (Millon, 1994; Millon, Davis, y Millon, 1997). Además, considera explícitamente, "evaluaciones para propósitos forenses" pues estos casos fueron considerados en la muestra normativa (Millon, 1994, p. 5).

Sobre su aplicabilidad en poblaciones forenses Archer, Vauter y Zoby (2006) señalan que el MCMI-III es utilizado en la evaluación de sujetos forenses siendo el segundo test más utilizado en temas civiles; además, tribunales lo han admitido para el testimonio en causas de custodia de niños, daño personal, violencia doméstica, determinación de discapacidad, y evaluación de simulación. Adicionalmente, el MCMI-III ofrece interpretaciones diseñadas para ambientes penitenciarios (Choca, 2010).
Aunque no se encontraron reportes específicos, el comportamiento psicométrico del MCMI-III en poblaciones forenses parece ser similar al encontrado en poblaciones clínicas. Al respecto, las cifras de protocolos válidos oscilan entre un $98 \%$ en el ámbito forense general (Rossi et al., 2007) y un $88 \%$ en el ámbito forensepenitenciario (Stoner, 2008). Los niveles de consistencia interna en muestras de sujetos clínicos y forenses (incluyendo presidiarios) alcanzan valores entre .67 y .88 para las escalas de personalidad y entre .70 y .90 para las escalas de síndromes clínicos (Rossi et al., 2007). Además, en prisioneros de Estados Unidos se han encontrado todos los patrones de personalidad descritos por la prueba con distintas tasas de prevalencia (ver Stoner, 2008) y, adicionalmente, se ha constatado que los sujetos reclusos que buscan la libertad condicional o una libertad temprana pueden exagerar su nivel de ajuste y no admitir los problemas conductuales que puedan tener (Bagby y Marshall, 2005).

En el ámbito de la validez diversas investigaciones han examinado la relación entre los trastornos de personalidad y las dimensiones del Modelo de los Cinco Factores (MCF; Costa y McCrae, 1985, 1990) utilizando una variedad de medidas y muestras que incluyen sujetos clínicos, estudiantes, adolescentes y adultos no clínicos (Saulsman y Page, 2004; Widiger y Costa, 2009). Estas investigaciones señalan que cada trastorno de personalidad del DSM-IV presenta asociaciones significativas y predecibles con el MCF que se expresan en las siguientes 14 relaciones entre las escalas de patrones de personalidad medidos por el MCMI-I, II y III, y las dimensiones del MCF: Esquizoide se asocia inversamente con Extroversión; Evitadora directamente con Neuroticismo e inversamente con Extroversión; Dependiente directamente con Neuroticismo; Histriónica directamente con Extroversión; Narcisista inversamente con Agradabilidad; Antisocial inversamente con 
Agradabilidad y con Ser Concienzudo; Compulsiva directamente con Ser Concienzudo; Esquizotípica directamente con Neuroticismo e inversamente con Extroversión; Limítrofe directamente con Neuroticismo; Paranoide directamente con Neuroticismo e inversamente con Agradabilidad. Estas relaciones pueden hacerse extensivas a agresores sexuales (Lehne, 2009; Madsen, Parsons, y Grubin, 2006).

Respecto de capacidad de discriminación, se ha reportado que sujetos forenses han obtenido mayor puntaje en prácticamente todas las escalas del MCMI-II respecto de sujetos no forenses (Cohen et al., 2002; Winberg y Vilalta, 2009).

\section{Problema de Investigación e Hipótesis}

El problema que aborda el presente estudio se refiere a las características psicométricas del MCML-III en población penitenciaria chilena. Aunque es un estudio exploratorio, pues no se han desarrollado investigaciones previas en Chile con este tipo de población, a la luz de los antecedentes recopilados en otros países es posible formular las siguientes hipótesis:

1. El MCMI-III presenta adecuada consistencia interna y estabilidad temporal.

2. Las escalas de personalidad del MCMI-III presentan adecuada validez concurrente con dimensiones del MCF medidas a través del NEO-FFI. Específicamente, las escalas de ambos instrumentos presentan entre sí, de un modo significativo, las 14 asociaciones antes mencionadas.

3. El MCMI-III presenta adecuada capacidad discriminante al contrastar la muestra penitenciaria con una muestra clínica, obteniendo puntajes mayores en las escalas del
MCMI-III aunque no es posible precisar especificamente en cuáles.

\section{MÉTODO}

\section{Participantes}

Sujetos varones adultos recluidos en el Complejo Penitenciario (CP) Alto Bonito de Puerto Montt, Chile $(n=123)$, que estaban cumpliendo condena por delitos del Titulo VII (Delitos Sexuales, tales como abuso sexual, abuso sexual infantil, estupro, violación, y violación de menor), Título VIII (Delitos contra las Personas, incluyendo amenazas, lesiones, homicidio frustrado, homicidio, y parricidio) y Título IX (Delitos contra la Propiedad, incluyendo daños, robo, robo con fuerza en las cosas, robo en lugar habitado, robo en bienes nacionales, robo por sorpresa, robo con intimidación, robo con violencia, e incendio) del Código Penal de Chile, y que asistían a la Escuela del CP.

Esta muestra intencionada cumplió con los siguientes criterios de inclusión: (a) ser varones chilenos; (b) cumpliendo condena; (c) edad entre 18 y 62 años; y (d) escolaridad $\geq$ Séptimo Básico. Los criterios de exclusión fueron: (a) sujetos varones imputados, (b) analfabetismo y/o escolaridad inferior a Séptimo Básico, (c) sujetos con problemas psiquiátricos recluidos en módulo especial del $\mathrm{CP}$, (d) empleados públicos y condenados por delitos de alta connotación pública, y (e) sujetos trabajadores de la empresa concesionaria en el CP.

\section{Procedimiento}

Se contactó a 350 sujetos, en grupos pequeños, en la Escuela del CP. Se les explicitó los objetivos del estudio y se les consultó si deseaban participar en forma voluntaria y anónima. Los 200 sujetos que aceptaron participar firmaron un Acta de Consentimiento Informado que les 
aseguraba la confidencialidad, resguardándose su identidad y la información proporcionada. A continuación completaron una Ficha de Registro y luego respondieron el MCMI-III y el NEO-FFI en una misma sesión. La aplicación se realizó en la sala de clases de la Escuela del CP, en grupos de 5 a 30 personas, en horario de clases; esta sala aseguraba buenas condiciones de iluminación, ventilación, control de distractores y era un espacio razonablemente tranquilo.

En la aplicación del MCMI-III, el investigador empleó algunos de los primeros ítems para asegurarse de que los participantes hubieran comprendido las instrucciones (Choca, 2010); el mismo procedimiento se utilizó para el NEO-FFI. Posteriormente, en un rango de 15 días y 3 meses, se aplicó el retest del MCMI-III a una submuestra de 30 sujetos.

De los 200 participantes, fueron eliminados quienes: a) respondieron en forma incompleta omitiendo al menos 12 ítems en cualquiera de los instrumentos; b) respondieron en la escala Sinceridad del MCMI-III fuera del rango aceptable (puntaje bruto $<34 \mathrm{y}>178$ ); c) tuvieron un protocolo inválido o cuestionable según la escala de Validez del MCMI-III. La muestra quedó conformada por 123 participantes y la submuestra para test-retest se redujo a 27 sujetos. Todos los análisis estadísticos se realizaron sobre los puntajes brutos del MCMI-III y del NEO-FFI, con el software SPSS versión 13.0.

\section{Instrumentos}

Ficha de Registro. Evaluó variables sociodemográficas, judiciales y psicológicas relevantes en el contexto forensepenitenciario. Recogió datos de procedencia urbana o rural, estado civil, presencia de hijos, nivel de escolaridad alcanzado, abandonos o expulsiones escolares, edad de inicio delictivo, cantidad de reclusiones, tiempo de encarcelamiento, etapa de la condena, consumo y abuso de alcohol y drogas y consumo de alcohol y/o drogas en la comisión del delito.

Inventario Clínico Multixial de Millon (MCMI)-III. Este instrumento contiene 175 ítems con respuesta Verdadero-Falso que se estructuran en 28 escalas: 11 escalas de patrones de personalidad básicos, 3 escalas de trastornos severos de personalidad, 7 escalas de síndromes clínicos básicos 0 moderados, 3 escalas de síndromes clínicos severos, 3 escalas modificadoras y 1 escala de validez.. Sus ítems se dividen en dos grupos: prototípicos, que representan características centrales de personalidad que son únicas para ese patrón o síndrome, y periféricos, que representan características no centrales y compartidas por dos o más patrones de personalidad 0 síndrome similares. Los ítems prototípicos son puntuados con el valor 2 , mientras que los ítems periféricos son puntuados con el valor 1 (Strack y Millon, 2007).

En este estudio se utilizó la versión chilena de Vinet et al. (1999). Esta versión, aplicada a una muestra clínica $(n=107)$ ha presentado niveles de consistencia interna similares a los obtenidos en muestras clínicas extranjeras, con alfas entre $.66 \mathrm{y}$ .86. Además, según Vinet et al. (1999), la matriz de correlaciones chilena MCMI-III / MMPI expresa, en lo esencial, el mismo patrón de correlaciones MCMI-III / MMPI-2 reportada por Millon (1994), constituyéndose en un indicador adecuado de la validez de la prueba en nuestro país.

NEO Five-Factor Inventory (NEO-FFI). Esta versión abreviada del NEO-PI (Costa y McCrae, 1985; 1989) está diseñada para evaluar, en sujetos entre 16 y 80 años de edad y con $6^{\circ}$ grado de escolaridad, las cinco dimensiones de personalidad que integran el MCF. El NEO-FFI contiene 60 ítems evaluados en una escala Likert con cinco opciones de respuesta, con puntuaciones entre 0 y 4 . La consistencia 
interna de las escalas del NEO-FFI ha oscilado entre .68 y .86 en estudios extranjeros; en muestras chilenas de estudiantes universitarios se han obtenido alfas entre .62 y .83 (Vinet, Fuentes, Melo, Muñoz, y Saiz, 1998). Este estudio empleó la versión chilena del NEO-FFI desarrollada por Vinet et al. (1998).

\section{RESULTADOS}

\section{Caracterización de la Muestra de Estudio}

La Tabla 1 presenta la caracterización sociodemográfica, psicológica y judicial de la muestra obtenida mediante la Ficha de Registro. La edad de los sujetos osciló entre 20 y 52 años $(M=28.54$, $D S=7.07$, Mediana $=27$ ) y fueron predominantemente urbanos $(91.1 \%)$ y solteros $(82.1 \%)$. Su nivel educacional osciló entre 7 y 14 años de educación formal $(M=8.78, D S=1.61$, Mediana $=8$ ), distribuyéndose desde enseñanza básica incompleta $(11,4 \%)$ hasta enseñanza superior completa con un máximo de 2 años de estudios superiores $(2.4 \%)$, predominando los sujetos con 8 años de escolaridad $(n=67 ; 54.5 \%)$.

TABLA 1.

Variables Sociodemográficas, Psicológicas y Judiciales de una Muestra de Reclusos Chilenos.

\begin{tabular}{|c|c|c|c|}
\hline Variable & & $f$ & $\%$ \\
\hline \multirow[t]{2}{*}{ Procedencia } & Urbana & 112 & 91.1 \\
\hline & Rural & 11 & 8.9 \\
\hline \multirow[t]{2}{*}{ Estado Civil } & Soltero & 101 & 82.1 \\
\hline & Casado & 22 & 17.9 \\
\hline \multirow[t]{2}{*}{ Hijos } & No & 42 & 34.1 \\
\hline & Sí & 81 & 65.9 \\
\hline \multirow[t]{4}{*}{ Categorías de Edad } & 18 a 24 años & 45 & 36.6 \\
\hline & 25 a 34 años & 56 & 45.5 \\
\hline & 35 a 44 años & 17 & 13.8 \\
\hline & 45 a 54 años & 5 & 4.1 \\
\hline \multirow[t]{3}{*}{ Abandonos / Expulsiones Escolares } & Nunca & 30 & 24.4 \\
\hline & $1 \mathrm{Vez}$ & 40 & 32.5 \\
\hline & Más de 1 Vez & 53 & 43.1 \\
\hline \multirow[t]{2}{*}{ Alcohol } & No & 97 & 78.9 \\
\hline & Sí & 26 & 21.1 \\
\hline \multirow[t]{2}{*}{ Drogas } & No & 71 & 57.7 \\
\hline & Sí & 52 & 42.3 \\
\hline \multirow[t]{4}{*}{ Tipos de Delitos } & Delitos Sexuales & 9 & 7.3 \\
\hline & Delitos contra las Personas & 21 & 17.1 \\
\hline & Delitos contra la Propiedad & 82 & 66.7 \\
\hline & Versatilidad Delictiva & 11 & 8.9 \\
\hline \multirow[t]{2}{*}{ Alcohol en Comisión de Delito } & No & 65 & 52.8 \\
\hline & Sí & 58 & 47.2 \\
\hline \multirow[t]{2}{*}{ Drogas en Comisión de Delito } & No & 61 & 49.6 \\
\hline & Sí & 62 & 50.4 \\
\hline \multirow[t]{3}{*}{ Etapa de la Reclusión } & Inicio & 45 & 36.6 \\
\hline & Mitad & 43 & 35.0 \\
\hline & Final & 35 & 28.5 \\
\hline
\end{tabular}


El $21.1 \%$ de los participantes autoreportó un consumo problemático de alcohol, mientras que el $42.3 \%$ autoreportó un consumo problemático de drogas. El $47.2 \%$ de los participantes manifestó haber ingerido alcohol y el $50.4 \%$ haber ingerido drogas en el momento de cometer el delito. Del total de la muestra, el $33.3 \%$ de los sujetos presentaba una reclusión, el 14.6\% presentaba 2 reclusiones, y el $52 \%$ más de 2 reclusiones. El tiempo de reclusión que presentaban los participantes fluctuó entre 5 y 175 meses $(M=38.36, D S=29.36$, Mediana $=30$ ) en tanto que la etapa de reclusión en la que se encontraban los distribuyó homogéneamente en los tres grupos.

Con respecto a los instrumentos utilizados, la prueba de Shapiro-Wilk indicó que mayoría de las escalas del MCMI-III presentaron una distribución no normal, lo cual era esperable pues según Strack y Millon (2007) por la naturaleza patológica de la mayoría de los ítems del MCMI, las distribuciones de puntajes tienden a no ser normales. La Tabla 2 presenta los estadísticos descriptivos de las escalas del MCMI-III en la muestra de estudio.

TABLA 2.

Estadísticos Descriptivos para las Escalas del MCMI-III en una Muestra de Reclusos Chilenos.

\begin{tabular}{|c|c|c|c|c|c|c|c|c|c|c|}
\hline \multicolumn{2}{|c|}{ Escalas del MCMI-III } & PMax & $M$ & DS & $M d n$ & Rango & Min & Max & Curt & Asim \\
\hline \multicolumn{11}{|c|}{ Escalas Modificadoras } \\
\hline$x$ & Sinceridad & 262.33 & 122.39 & 25.96 & 123.67 & 115.67 & 59.67 & 175.33 & -.48 & -.18 \\
\hline Y & Deseabilidad & 21 & 14.57 & 3.42 & 15 & 17 & 3 & 20 & .36 & -.81 \\
\hline Z & Alteración & 33 & 11.18 & 6.68 & 11 & 30 & 0 & 30 & -.62 & .36 \\
\hline \multicolumn{11}{|c|}{ Escalas Básicas de Personalidad } \\
\hline 1 & Esquizoide & 23 & 10.65 & 3.97 & 11 & 17 & 2 & 19 & -.58 & -.06 \\
\hline $2 \mathrm{~A}$ & Evitadora & 24 & 8.67 & 4.67 & 8 & 22 & 1 & 23 & -.02 & .59 \\
\hline $2 \mathrm{~B}$ & Depresiva & 23 & 9.46 & 5.09 & 10 & 22 & 0 & 22 & -.70 & .22 \\
\hline 3 & Dependiente & 24 & 7.94 & 4.11 & 8 & 18 & 0 & 18 & -.77 & .32 \\
\hline 4 & Histriónica & 24 & 14.72 & 4.03 & 15 & 18 & 4 & 22 & -.20 & -.58 \\
\hline 5 & Narcisista & 32 & 17.97 & 4.11 & 18 & 22 & 4 & 26 & .58 & -.53 \\
\hline $6 \mathrm{~A}$ & Antisocial & 24 & 13.88 & 4.98 & 15 & 21 & 2 & 23 & -.68 & -.39 \\
\hline $6 B$ & Agresivo-Sádica & 27 & 13.02 & 5.88 & 12 & 26 & 1 & 27 & -.68 & .05 \\
\hline 7 & Compulsiva & 25 & 15.16 & 4.32 & 15 & 18 & 6 & 24 & -.67 & -.13 \\
\hline $8 \mathrm{~A}$ & Pasivo-Agresiva & 25 & 10.82 & 5.02 & 11 & 22 & 0 & 22 & -.52 & -.10 \\
\hline $8 \mathrm{~B}$ & Autodestructiva & 22 & 6.08 & 3.82 & 6 & 15 & 0 & 15 & -.69 & .33 \\
\hline \multicolumn{11}{|c|}{ Escalas Severas de Personalidad } \\
\hline S & Esquizotípica & 25 & 10.18 & 5.79 & 10 & 24 & 0 & 24 & -.71 & .27 \\
\hline C & Limítrofe & 25 & 8.44 & 4.72 & 9 & 20 & 0 & 20 & -.81 & .19 \\
\hline$P$ & Paranoide & 26 & 12.54 & 5.43 & 13 & 25 & 0 & 25 & -.51 & -.19 \\
\hline \multicolumn{11}{|c|}{ Síndromes Clínicos Moderados } \\
\hline A & Ansiedad & 20 & 7.89 & 4.80 & 8 & 19 & 0 & 19 & -.88 & .15 \\
\hline $\mathrm{H}$ & Histeriforme & 17 & 5.44 & 3.88 & 5 & 16 & 0 & 16 & -.71 & .45 \\
\hline $\mathrm{N}$ & Hipomanía & 18 & 8.86 & 3.71 & 9 & 16 & 1 & 17 & -.63 & .18 \\
\hline D & Neurosis Depresiva & 20 & 6.02 & 4.22 & 5 & 18 & 0 & 18 & -.50 & .58 \\
\hline B & Abuso de Alcohol & 21 & 8.78 & 4.18 & 8 & 19 & 0 & 19 & -.22 & .39 \\
\hline $\mathrm{T}$ & Abuso de Drogas & 20 & 11.70 & 5.17 & 12 & 19 & 1 & 20 & -1.01 & -.25 \\
\hline $\mathrm{R}$ & D. de Estrés Post-Traum. & 21 & 6.57 & 5.12 & 5 & 21 & 0 & 21 & -.45 & .69 \\
\hline \multicolumn{11}{|c|}{ Síndromes Clínicos Severos } \\
\hline SS & Pensamiento Psicótico & 23 & 9.23 & 5.10 & 10 & 29 & 0 & 20 & -.83 & .06 \\
\hline $\mathrm{CC}$ & Depresión Mayor & 23 & 6.98 & 5.30 & 6 & 21 & 0 & 21 & -.53 & .56 \\
\hline PP & Delirio Psicótico & 17 & 5.55 & 3.35 & 5 & 15 & 0 & 15 & -.17 & .56 \\
\hline
\end{tabular}

Nota. PMax = Puntaje máximo posible. Mdn = Mediana. 
En el caso del NEO-FFI, los reclusos tendieron a presentar puntajes en torno a la media en Neuroticismo $(M=20.03)$, Apertura a la Experiencia $(M=25.20)$ y Agradabilidad $(M=25.78)$, puntajes levemente altos en Extroversión $(M=28,33)$ $y$ altos en Ser Concienzudos $(M=32.38)$.

\section{Confiabilidad}

El cálculo de la consistencia interna entregó valores bajos a moderados para la mayoría de las escalas del MCMI-III. En las escalas de personalidad, el rango varía entre .78 (personalidad Esquizotípica) y .40 (personalidad Narcisista), mientras que en las escalas de síndromes clínicos, el rango oscila entre .82 (Desorden de Estrés Postraumático) y .64 (Hipomanía y Delirio Psicótico). Tal como se aprecia en la Tabla 3 , solo 15 escalas (58\%) obtuvieron un alfa $\geq .70$, criterio mínimo para considerar un nivel de consistencia interna adecuado (Nunnally, 1978). Se observó, además, que los mayores niveles de consistencia interna se presentan en las escalas de síndromes clínicos, con excepción de las escalas Hipomanía y Delirio Psicótico.

TABLA 3.

Confiabilidad por Consistencia Interna y Estabilidad Temporal de las Escalas del MCMI-III.

\begin{tabular}{|c|c|c|c|c|c|c|}
\hline \multirow{2}{*}{\multicolumn{2}{|c|}{ Escalas del MCMI-III }} & \multicolumn{3}{|c|}{ Alfa de Cronbach } & \multicolumn{2}{|c|}{ Test - retest } \\
\hline & & $\begin{array}{c}\text { Presente } \\
\text { Estudio }\end{array}$ & $\begin{array}{c}\text { Vinet et al. } \\
\text { (1999) }\end{array}$ & $\begin{array}{l}\text { Millon } \\
\text { (1994) }\end{array}$ & $\begin{array}{c}\text { Presente } \\
\text { Estudio } \\
\end{array}$ & $\begin{array}{l}\text { Millon } \\
\text { (1994) }\end{array}$ \\
\hline \multicolumn{7}{|c|}{ Escalas Modificadoras } \\
\hline$x$ & Sinceridad & & & & $.77^{\star * \star}$ & .94 \\
\hline Y & Deseabilidad (21) & .69 & .77 & .86 & $.39^{*}$ & .92 \\
\hline Z & Alteración (33) & .87 & .92 & .95 & $.61^{* \star *}$ & .82 \\
\hline \multicolumn{7}{|c|}{ Escalas Básicas de Personalidad } \\
\hline 1 & Esquizoide (16) & .60 & .72 & .81 & $.69^{* * *}$ & .89 \\
\hline $2 \mathrm{~A}$ & Evitadora (16) & .68 & .84 & .89 & $.48^{* *}$ & .89 \\
\hline $2 \mathrm{~B}$ & Depresiva (15) & .73 & .84 & .89 & $.58^{\star \star *}$ & .93 \\
\hline 3 & Dependiente (16) & .62 & .79 & .85 & $.40^{*}$ & .89 \\
\hline 4 & Histriónica (17) & .60 & .80 & .81 & $.65^{\star \star \star}$ & .91 \\
\hline 5 & Narcisista (24) & .40 & .69 & .67 & .31 & .89 \\
\hline $6 \mathrm{~A}$ & Antisocial (17) & .72 & .73 & .77 & $.72^{\star * *}$ & .93 \\
\hline $6 \mathrm{~B}$ & Agresivo-Sádica (20) & .77 & .80 & .79 & $.70^{\star \star \star}$ & .88 \\
\hline 7 & Compulsiva (17) & .63 & .66 & .66 & $.69^{* * *}$ & .92 \\
\hline $8 \mathrm{~A}$ & Pasivo-Agresiva (16) & .68 & .80 & .83 & $.79^{\star \star \star}$ & .89 \\
\hline $8 \mathrm{~B}$ & Autodestructiva (15) & .59 & .78 & .87 & $.49^{* *}$ & .91 \\
\hline \multicolumn{7}{|c|}{ Escalas Severas de Personalidad } \\
\hline$S$ & Esquizotípica (16) & .78 & .85 & .85 & $.65^{\star \star *}$ & .87 \\
\hline C & Limítrofe (16) & .71 & .77 & .85 & $.56^{\star * *}$ & .93 \\
\hline$P$ & Paranoide (17) & .70 & .83 & .84 & $.62^{\star \star \star}$ & .85 \\
\hline \multicolumn{7}{|c|}{ Síndromes Clínicos Moderados } \\
\hline$A$ & Ansiedad (14) & .77 & .82 & .86 & $.66^{* * *}$ & .84 \\
\hline $\mathrm{H}$ & Histeriforme (12) & .70 & .82 & .86 & $.52^{* *}$ & .96 \\
\hline $\mathrm{N}$ & Hipomanía (13) & .64 & .76 & .71 & $.58^{\star * *}$ & .93 \\
\hline $\mathrm{D}$ & Neurosis Depresiva (14) & .71 & .84 & .88 & $.43^{*}$ & .91 \\
\hline B & Abuso de Alcohol (15) & .70 & .75 & .82 & $.75^{\star \star \star}$ & .92 \\
\hline $\mathrm{T}$ & Abuso de Drogas (14) & .77 & .70 & .83 & $.77^{* * *}$ & .91 \\
\hline $\mathrm{R}$ & D. de Estrés P-Traumático (16) & .82 & .86 & .89 & $.48^{* *}$ & .94 \\
\hline \multicolumn{7}{|c|}{ Sindromes Clínicos Severos } \\
\hline SS & Pensamiento Psicótico (17) & .77 & .85 & .87 & $.65^{\star \star \star}$ & .92 \\
\hline & Depresión Mayor (17) & .80 & .86 & .90 & $.44^{*}$ & .95 \\
\hline & Delirio Psicótico (13) & .64 & .77 & .79 & $.72^{\star * *}$ & .86 \\
\hline
\end{tabular}

Nota. No se reportan valores Alfa para la escala Sinceridad (cuyo valor se deriva de puntuaciones de las escalas básicas de personalidad) ni para la escala de Validez (que está conformada por sólo 3 ítems).

Los valores Alfa $\geq .70$ se muestran en negrita. La submuestra para el test - retest fue de 27 participantes.

${ }^{*} p<.05,{ }^{* *} p<.01,{ }^{* * *} p<.001$. 
Respecto de estabilidad temporal, es posible apreciar en la Tabla 3 que las correlaciones significativas test-retest de los puntajes de los reclusos chilenos son, respecto de las correlaciones obtenidas por Millon (1994), similares en cantidad pero bastante menores en intensidad, oscilando entre .39 (Deseabilidad) y .79 (PasivoAgresiva). Por otra parte, aunque se esperaría que la estabilidad de las escalas de personalidad fuese más alta que la de las escalas de síndromes clínicos (Choca, 2010), en este caso las diferencias son muy leves.

Adicionalmente, se calculó la confiabilidad por consistencia interna de las dimensiones del NEO-FFI. Con excepción de la escala Ser Concienzudo que obtuvo un alfa $=.74$, las otras escalas del NEO-FFI obtuvieron alfas $\leq .70$, a saber: Neuroticismo $=.51$, Extroversión $=.56$, Apertura a la Experiencia $=.14 \mathrm{y}$ Agradabilidad $=51$.

\section{Validez}

Primeramente, para examinar la validez del MCMI-III se generó, una matriz de correlaciones $r$ de Pearson entre las 11 escalas de patrones de la personalidad del MCMI-III y las 5 dimensiones del NEO-FFI. Esta matriz se presenta en la Tabla 4. Para la prueba de hipótesis se estableció un nivel de .001 como criterio de significancia (corrección de Bonferroni para 55 correlaciones) junto con un criterio de magnitud de la correlación $\geq .30$.

TABLA 4.

Correlaciones entre las Escalas del MCMI-III y NEO-FFI en una Muestra de Reclusos Chilenos.

\begin{tabular}{|c|c|c|c|c|c|c|}
\hline \multirow{2}{*}{\multicolumn{2}{|c|}{$\begin{array}{l}\text { Escalas de Personalidad } \\
\text { del MCMI-III }\end{array}$}} & \multicolumn{5}{|c|}{ Escalas NEO-FFI } \\
\hline & & $\mathrm{N}$ & $E$ & $\mathrm{AE}$ & A & $\mathrm{SC}$ \\
\hline 1 & Esquizoide & $.16^{*}$ & $-.41^{\star \star *}$ & $-.15^{*}$ & $-.26^{* *}$ & $-.19^{\star}$ \\
\hline $2 \mathrm{~A}$ & Evitadora & $.30^{* * *}$ & $-.27^{\star \star *}$ & -.14 & -.04 & $-.21^{*}$ \\
\hline 3 & Dependiente & $.26^{\star \star}$ & $-.11^{*}$ & $-.17^{*}$ & .12 & $-.16^{*}$ \\
\hline 4 & Histriónica & -.10 & $.47^{\star \star *}$ & $.18^{*}$ & .07 & .09 \\
\hline 5 & Narcisista & -.06 & $.16^{*}$ & .06 & $-18^{*}$ & .03 \\
\hline $6 \mathrm{~A}$ & Antisocial & $.29^{\star \star *}$ & -.07 & .03 & $-.36^{\star \star *}$ & $-.26^{* *}$ \\
\hline 7 & Compulsiva & -.12 & .11 & -.00 & $29^{* * *}$ & $.36^{* * *}$ \\
\hline $8 \mathrm{~A}$ & Pasivo-Agresiva & $.33^{* \star *}$ & $-.18^{*}$ & -.09 & $-.29^{* * *}$ & $-.27^{\star \star}$ \\
\hline S & Esquizotípica & $.26^{* \star}$ & $-.25^{* *}$ & -.08 & $-.22^{* *}$ & $-.22^{* *}$ \\
\hline C & Limítrofe & $.35^{* * *}$ & $-.25^{* *}$ & -.14 & $-.21^{* *}$ & $-.25^{* *}$ \\
\hline P & Paranoide & $.31^{\star \star *}$ & $-.20^{*}$ & -.03 & $-.27^{* \star *}$ & $-.15^{\star}$ \\
\hline
\end{tabular}

Nota. $\mathrm{N}$ = Neuroticismo; $\mathrm{E}$ = Extroversión; $\mathrm{AE}=$ Apertura a la Experiencia; $\mathrm{A}$ = Agradabilidad; $\mathrm{SC}=$ Ser Concienzudo Se muestran valores $p$ para las asociaciones indicadas por la teoría e investigación, así como para aquellas que, no siendo indicadas en la literatura, resultaron significativas. Las asociaciones que fueron hipotetizadas se indican en negrita. Sig. de 1 cola para asociaciones hipotetizadas según la literatura. ${ }^{*} p<.05,{ }^{* *} p<.01,{ }^{* * *} p<.001$. 
Aunque en la matriz obtenida aparecen todas las asociaciones hipotetizadas (y varias otras), con los criterios establecidos se aceptan siete de las 14 asociaciones hipotetizadas, a saber: Esquizoide correlacionó inversamente con Extroversión $(r=-.41, p<.001)$; Evitadora correlacionó directamente con Neuroticismo $(r=.30, p<$ .001); Histriónica directamente con
Extroversión ( $r=.47, p<.001)$; Antisocial inversamente con Agradabilidad $(r=-.36, p$ $<.001$ ); Compulsiva directamente con Ser Concienzudo $(r=.36, p<.001)$; Limítrofe directamente con Neuroticismo $(r=.35, p<$ $.001) ;$ y Paranoide directamente con Neuroticismo $(r=.31, p<.001)$. Estos resultados muestran una moderada relación empírica entre ambos instrumentos.

TABLA 5.

Comparación entre Muestra Penitenciaria y una Muestra Clínica en las Escalas del MCMI-III.

\begin{tabular}{|c|c|c|c|c|c|c|c|}
\hline \multirow{2}{*}{\multicolumn{2}{|c|}{ Escalas del MCMI-III }} & \multicolumn{2}{|c|}{$\begin{array}{l}\text { Muestra Clínica } \\
\quad(n=125)\end{array}$} & \multicolumn{2}{|c|}{$\begin{array}{l}\text { Muestra Penitenciaria } \\
\qquad(n=123)\end{array}$} & \multirow[b]{2}{*}{$t$} & \multirow[b]{2}{*}{$d$} \\
\hline & & $M$ & $(D S)$ & $M$ & (DS) & & \\
\hline \multicolumn{8}{|c|}{ Escalas Modificadoras } \\
\hline Y & Deseabilidad & 11.50 & $(4.31)$ & 14.57 & $(3.42)$ & $6,22^{\star * *}$ & 0,79 \\
\hline Z & Índice de Alteración & 14.73 & $(8.26)$ & 11.18 & $(6.68)$ & $3,72^{\star * *}$ & 0,47 \\
\hline \multicolumn{8}{|c|}{ Escalas Básicas de Personalidad } \\
\hline 1 & Esquizoide & 6.98 & (3.35) & 10.65 & $(3.97)$ & $7,86^{\star * *}$ & 0,99 \\
\hline $2 \mathrm{~A}$ & Evitadora & 6.90 & $(4.11)$ & 8.67 & $(4.67)$ & $3,17^{* *}$ & 0,40 \\
\hline $2 \mathrm{~B}$ & Depresiva & 7.22 & (3.96) & 9.46 & $(5.09)$ & $3,86^{\star * *}$ & 0,49 \\
\hline 3 & Dependiente & 6.61 & (3.63) & 7.94 & $(4.11)$ & $2,70^{* *}$ & 0,34 \\
\hline 4 & Histriónica & 8.33 & $(4.07)$ & 14.72 & $(4.03)$ & $12,42^{\star * \star}$ & 1,57 \\
\hline 5 & Narcisista & 11.04 & $(4.04)$ & 17.97 & $(4.11)$ & $13,39^{* * *}$ & 1,70 \\
\hline $6 \mathrm{~A}$ & Antisocial & 6.31 & (3.25) & 13.88 & $(4.98)$ & $14,15^{\star * *}$ & 1,79 \\
\hline $6 B$ & Agresivo-Sádica & 8.81 & $(4.27)$ & 13.02 & $(5.88)$ & $6,44^{* * *}$ & 1,00 \\
\hline 7 & Compulsiva & 9.14 & (3.21) & 15.16 & $(4.32)$ & $12,44^{* \star *}$ & 1,58 \\
\hline $8 \mathrm{~A}$ & Pasivo-Agresiva & 7.64 & $(3.86)$ & 10.82 & $(5.02)$ & $5,59^{* * *}$ & 0,71 \\
\hline $8 \mathrm{~B}$ & Autodestructiva & 4.98 & $(3.42)$ & 6.08 & $(3.82)$ & $2,39^{*}$ & 0,30 \\
\hline \multicolumn{8}{|c|}{ Escalas Severas de Personalidad } \\
\hline S & Esquizotípica & 5.46 & $(4.17)$ & 10.18 & $(5.79)$ & $7,36^{\star * *}$ & 0,93 \\
\hline C & Limítrofe & 6.30 & $(3.55)$ & 8.44 & $(4.72)$ & $4,03^{\star * *}$ & 0,51 \\
\hline$P$ & Paranoide & 6.31 & $(4.13)$ & 12.54 & $(5.43)$ & $10,16^{* * *}$ & 1,28 \\
\hline \multicolumn{8}{|c|}{ Síndromes Clínicos Moderados } \\
\hline$A$ & Ansiedad & 6.62 & $(3.70)$ & 7.89 & $(4.80)$ & $2,33^{*}$ & 0,29 \\
\hline $\mathrm{H}$ & Histeriforme & 5.03 & $(3.24)$ & 5.44 & $(3.88)$ & 0,90 & 0,11 \\
\hline $\mathrm{N}$ & Hipomanía & 6.06 & (3.18) & 8.86 & (3.71) & $6,38^{* * *}$ & 0,81 \\
\hline D & Neurosis Depresiva & 5.97 & (3.89) & 6.02 & $(4.22)$ & 0,10 & 0,01 \\
\hline B & Abuso de Alcohol & 4.94 & (3.03) & 8.78 & $(4.18)$ & $8,27^{\star * *}$ & 1,05 \\
\hline $\mathrm{T}$ & Abuso de Drogas & 3.80 & $(2.45)$ & 11.70 & $(5.17)$ & $15,34^{* * *}$ & 1,94 \\
\hline $\mathrm{R}$ & D. de Estrés P-Traum. & 6.34 & $(4.24)$ & 6.57 & $(5.12)$ & 0,38 & 0,05 \\
\hline \multicolumn{8}{|c|}{ Síndromes Clínicos Severos } \\
\hline SS & Pensamiento Psicótico & 6.82 & $(4.30)$ & 9.23 & $(5.10)$ & $4,02^{\star \star \star}$ & 0,51 \\
\hline $\mathrm{CC}$ & Depresión Mayor & 6.93 & $(4.47)$ & 6.98 & $(5.30)$ & 0,08 & 0,01 \\
\hline PP & Delirio Psicótico & 3.22 & $(2.85)$ & 5.55 & $(3.35)$ & $5,90^{\star * *}$ & 0,75 \\
\hline
\end{tabular}


En segundo lugar, para determinar la capacidad del MCMI-III para diferenciar los perfiles de la muestra penitenciaria, se compararon los puntajes obtenidos por estos sujetos con los puntajes obtenidos en la muestra clínica chilena (Vinet et al., 1999) a través de una prueba $t$ para muestras independientes, realizando 26 comparaciones de escalas entre ambos grupos. Para establecer resultados sustantivos más allá del dato estadístico, se consideró que la corrección de Bonferroni arroja un $p>.002$ como criterio de significancia y, además, se calculó el tamaño de la diferencia ( $d$ de Cohen). Como se observa en la Tabla 5 , los reclusos presentaron puntajes mayores que los sujetos clínicos en todas las escalas de personalidad con $d \geq 0.30$ y en cinco de las 10 escalas de síndromes clínicos. Llama la atención el único puntaje significativamente menor, obtenido en la escala modificadora Índice de Alteración, que unido a la alta Deseabilidad indicaría que los sujetos recluidos presentan una menor tendencia que los sujetos clínicos de aparecer mal psicológicamente junto a un mayor deseo de aparecer socialmente atractivos, moralmente virtuosos, o emocionalmente bien compuestos. Estos resultados son sólo orientadores de lo que podría encontrarse realmente al comparar sujetos forenses y clínicos pues ambas muestras no son estrictamente comparables dado que los resultados de la muestra clínica utilizada corresponden a hombres y mujeres.

\section{DISCUSIÓN}

El propósito de este estudio fue evaluar las propiedades psicométricas del MCMI-III en Chile en población penitenciaria adulta masculina. Los resultados obtenidos señalan que el MCMI-III entrega medidas sólo medianamente consistentes y estables, no comparables a las obtenidas en sujetos clínicos chilenos (Vinet et al., 1999) ni en Estados Unidos (Millon, 1994) con el instrumento original; por otro lado, la obtención de correlaciones significativas con magnitudes moderadas en la dirección esperada, apoya parcialmente la concurrencia entre el MCMI-III y el MCF en la evaluación de trastornos de la personalidad; por último, las diferencias significativas entre reclusos y sujetos clínicos en la mayoría de las escalas del MCMI-III sugieren que el instrumento podría discriminar adecuadamente entre estas dos poblaciones.

Al examinar, en detalle, los resultados de consistencia interna se aprecia que bajos niveles de fiabilidad se han presentado también en muestras extranjeras de sujetos psiquiátricos (Haddy, Strack, y Choca, 2005) y en sujetos clínicos y forenses (Rossi et al., 2007) en escalas similares. Además, tal como lo plantea Choca (2010), la baja consistencia interna de las escalas básicas de personalidad, que evalúan estilos de personalidad antes que psicopatología, podría estar relacionado a que, en su esfuerzo adaptativo por diferenciarse del resto, los reclusos desplieguen conductas diversas y contradictorias pues, tal como lo señala Bermúdez-Fernández (2006, p. 360) "hace falta una posición, una opción personal, un criterio actitudinal y conductual sobre el que construir una situación propia que permita la supervivencia en una situación carcelaria".

Adicionalmente, la consistencia interna puede haber sido afectada por variables como la motivación y el oposicionismo: Se debe considerar que una alta tasa de reclusos $(43 \%)$ rechazó participar del estudio; que hubo una alta tasa de protocolos cuestionables (21\%) caracterizados por respuesta azarosa y/o comportamiento negativista extremo; que la tasa de protocolos válidos (75\%) es más baja que la obtenida por reclusos estadounidenses (Stoner, 2008); y que los valores alfa más altos se obtienen en el subgrupo de sujetos en etapa final de la reclusión. Por otro lado, Kunst y Hoyer (2003) advertían sobre valores alfa bajos en tres escalas del NEO-FFI, en una muestra 
de ofensores. Así, la modesta consistencia interna del MCMI-III podría no ser atribuible al instrumento per se sino que al tipo de muestra (sujetos recluidos) con sus características particulares (BermúdezFernández, 2006). Por último es necesario señalar que las confiabilidades obtenidas en este estudio son un dato importante para la validez pues "la confiabilidad de una medida limita cuán fuertemente esa medida puede correlacionar con otra variable" (John y Soto, 2007, p. 464).

La matriz de correlaciones MCMI-III /NEO-FFI muestra que aunque algunas correlaciones hipotetizadas no emergieron en los niveles previstos, apareció un patrón claro de asociaciones significativas, en la dirección esperada, entre patrones de personalidad y dimensiones del MCF, que está en línea con los antecedentes teóricos y empíricos, y que apoya una moderada validez concurrente entre MCMI-III y el MCF.

Respecto de asociaciones no hipotetizadas, destacaron las correlaciones de la escala Pasivo-Agresiva con Neuroticismo (directa) y Agradabilidad y Ser Concienzudo (inversas); si bien la primera de estas asociaciones encuentra sustento empírico solo en el estudio de Costa y McCrae (1990), la combinación de baja Agradabilidad y bajo Ser Concienzudo es característico del patrón pasivo-agresivo (Widiger, Trull, Clarkin, Sanderson, y Costa, 2009). Finalmente, llama la atención la asociación directa entre la escala Antisocial y Neuroticismo que indicaría que en los reclusos chilenos la tendencia Antisocial aparece asociada a un desajuste emocional, mereciendo estudios posteriores.

En relación a la comparación del grupo de reclusos con la muestra clínica de Vinet et al. (1999), es importante señalar que este es el único estudio chileno que permite una comparación y que las diferencias encontradas deberán ser corroboradas en estudios posteriores, no permitiendo la comparación actual establecer relaciones específicas dada la no equivalencia de las muestras en una variable tan importante como el sexo de los sujetos.

Al decantar los resultados expuestos y comentados en los párrafos precedentes, en este estudio se puede concluir lo siguiente: La consistencia interna del MCMI-III en reclusos chilenos puede ser afectada por variables como motivación y oposicionismo dependiendo de la etapa del encarcelamiento en que ellos se encuentren. Por otro lado, la deseabilidad social habría influido en los puntajes de las escalas del MCMI-III y del NEO-FFI. Los resultados de estabilidad temporal son poco confiables pues a las condiciones previas se agrega la gran variabilidad en el rango de tiempo empleado entre test y retest. La baja consistencia interna del MCMI-III y del NEOFFI probablemente atenuaron las asociaciones del Modelo de Millon con el MCF. Pese a estas condiciones el MCMI-III correlacionó con el NEO-FFI en la forma esperada. Las escalas del MCMI-III se asociaron más significativamente a los factores Neuroticismo y Extroversión del MCF; este dato coincide con las relaciones entre trastornos de la personalidad y MCF encontradas en los estudios de Costa y McCrae (1990, citado por Lehne, 2009) y de Lehne (2009). Aunque Agradabilidad apareció asociada a los patrones de personalidad en magnitudes más bajas, su asociación es consistente pues Agradabilidad aparece como un factor importante cuando se evalúa conducta antisocial y agresión (Jones, Miller, y Lynam, 2011). Finalmente, el MCMI-III discriminó coherentemente a sujetos forenses-penitenciarios de sujetos clínicos en varias de sus escalas aun considerando las limitaciones comparativas por la diferente composición de las muestras en una variable tan relevante como sexo.

Considerando que este estudio fue exploratorio y local, los resultados sugieren que el MCMI-III presenta una moderada validez concurrente con el MCF y una 
moderada capacidad discriminante. Debido a la insuficiente confiabilidad obtenida, estos resultados son solo orientadores de lo que puede esperarse en población penitenciaria. Aunque se requiere trabajo adicional para evaluar sus propiedades psicométricas en poblaciones forenses, se plantea que el MCMI-III puede ser un instrumento útil para evaluar personalidad y síndromes clínicos en reclusos chilenos, para comprender el funcionamiento psicológico detrás de la conducta delictiva; este instrumento permitiría, en base a un diagnóstico forense, la visualización de estrategias de intervención conductual más efectivas dentro de las cárceles.

A la luz de los resultados, se sugiere algunos resguardos para la aplicación y uso del MCMI-III en poblaciones forensespenitenciarias: (a) examinar en términos clínico-forenses la capacidad de lectura del sujeto; (b) evaluar la disposición a responder el instrumento en forma adecuada; (c) aplicar el instrumento en dependencias con acceso restringido, con apropiada luz, y libre de ruidos y/o distracciones; (d) chequear las respuestas al instrumento mientras es respondido, en orden a detectar omisiones, respuestas al azar, y respuestas incongruentes.

Entre las fortalezas de este estudio, dado que es el primero del MCMI-III en sujetos reclusos chilenos, se puede señalar que aportó una "base" para estudios futuros con el MCMI-III en poblaciones forenses adultas. Entre sus limitaciones está el hecho de que trabajó con población penal local, poco generalizable por su especificidad; además, utilizó en sus contrastaciones con sujetos clínicos al único estudio reportado a nivel nacional sin poder disponer de los datos originales. Finalmente, este estudio fue exploratorio y local y sus resultados no son definitivos ni generalizables en la población penitenciaria.

Futuras líneas de investigación con el MCMI-III podrán examinar la relación entre patrones de personalidad y facetas de las dimensiones del MCF; examinar diferencias en patrones de personalidad entre sujetos recluidos masculinos y femeninos; analizar la relación entre patrones de personalidad y tipos de delitos; examinar las características psicométricas del MCMI-III en muestras más grandes de reclusos así como en otras poblaciones forenses. 
REFERENCIAS

American Psychiatric Association (1987). Diagnostic and Statistical Manual of Mental Disorders (3th ed.), DSM-III-R. Washington: American Psychiatric Association.

American Psychiatric Association (2000). Diagnostic and Statistical Manual of Mental Disorders (4th ed.), DSM-IV-TR. Washington: American Psychiatric Association.

Archer, R., Buffington-Vollum, J., Vauter, R., y Handel, R. (2006). A survey of psychological test use among forensic psychologists. Journal of Personality Assessment, 87(1), 84 - 89.

Archer, R., Vauter, R., y Zoby, M. (2006). Introduction to forensic uses of clinical assessment instruments. En R. Archer (Ed.), Forensic Uses of Clinical Assessment Instruments (pp. 1-18). New Jersey: Lawrence Erlbaum Associates.

Bagby, R. y Marshall, M. (2005). Assessing response bias with the MCMI modifying indices. En R. Craig (Ed.), New Directions in Interpreting the Millon Clinical Multiaxial Inventory-III (pp. 227247). New Jersey: Wiley.

Bennet, T., Holloway, K, y Farrington, D. (2008). The statistical association between drug misuse and crime: $A$ meta-analysis. Aggression and Violent Behavior, 13, 107-118.

Bermúdez-Fernández, J. (2006). Efectos psicológicos del encarcelamiento. En J. Sierra, E.Jiménez y G. Buela-Casal (Eds.), Psicología Forense: Manual de Técnicas y Aplicaciones (pp. 348-371). Madrid: Biblioteca Nueva.

Boden, J., Fergusson, D., y Horwood, J. (2012). Alcohol misuse and criminal offending: Findings from a 30-year longitudinal study. Drug and Alcohol Dependence,

http://dx.doi.org/10.1016/j.drugalcdep.2 012.07.014

Cardenal, V., Sánchez, M., y Ortiz-Tallo, M. (2007). Los trastornos de personalidad según el modelo de Millon: una propuesta integradora. Clínica y Salud, 18(3): 305-324.

Choca, J. (2010). Interpretive Guide to the Millon Clinical Multiaxial Inventory (3rd ed.) Washington: American Psychological Association.

Cohen, L., Watras, S., McGeoch, P., Poznansky, O., Itskovich, Y., Murphy, S., Klein, E., Cullen, K., y Galynker, I. (2002). Impulsive personality traits in male pedophiles versus healthy controls: Is pedophilia an impulsiveaggressive disorder? Comprehensive Psychiatry, 43(12), 127-134.

Cooper, D. (1994). Delincuencia común en Chile. Santiago: LOM Ediciones.

Costa, P. y McCrae, R. (1985). The NEO Personality Inventory Manual. Odessa: Psychological Assessment Resources.

Costa, P. y McCrae, R. (1989). The NEOPI/NEO-FFI manual supplement. Odessa: Psychological Assessment Resources.

Costa, P. y McCrae, R. (1990). Personality disorders and the five-factor model of personality. Journal of Personality Disorders, 4, 362-371.

Dyer, F. (2008). Using the Millon inventories in forensic psychology. En T. Millon y C. Bloom (Eds.), The Millon Inventories (2nd ed., pp. 177-198). New York: Guilford

Fernández-Montalvo, J. y Echeburúa, E. (2008). Trastornos de personalidad y psicopatía en hombres condenados por violencia grave contra la pareja. Psicothema, 20(2), 193 - 198.

Haddy, C., Strack, S., y Choca, J. (2005). Linking personality disorders and clinical syndromes on the MCMI-III. Journal of Personality Assessment, 84(2), 193-204.

Heilbrun, K., Marczyk, G., DeMatteo, D., Mack-Allen, J. (2007). A principlesbased approach to forensic mental health assessment: Utility and update. En A. Goldstein (Ed.), Forensic Psychology. New Jersey: John Wiley y Sons. 
John, O., y Soto, C. (2007). The importance of being valid: Reliability and the process of construct validation. En $\mathrm{R}$. Robins, R. Fraley, y R. Krueger (Eds.), Handbook of Research Methods in Personality Psychology (pp. 461- 494). New York: Guilford.

Jones, S., Miller, J., y Lynam, D. (2011). Personality, antisocial behavior, and aggression: A meta-analytic review. Journal of Criminal Justice. doi: 10.1016/j.jcrimjus.2011.03.004

Kline, P. (2000). Handbook of Psychological Testing (2nd ed.). London: Routledge.

Kunst, H. y Hoyer, J. (2003). Psychometrische analysen von IIP und NEO-FFI bei Straftätern Diagnostica, 49, 1, 24-33.

Lehne, G. (2009). The NEO Personality Inventory and the Millon Clinical Multiaxial Inventory in the forensic evaluation of sex offenders. En P.Costa y T. Widiger (Eds.), Personality Disorders and the Five-factor Model of Personality (2nd ed., pp. 269-282). Washington: American Psychological Association.

Madsen, L., Parsons, S., y Grubin, D. (2006). The relationship between the five-factor model and DSM personality disorder in a sample of child molesters. Personality and Individual Differences, 40, 227-236.

Millon, T. (1994). Millon Clinical Multiaxial Inventory-III (MCMI-III) Manual. Minneapolis: National Computer Systems.

Millon, T. y Davis, R. (1998). Trastornos de Personalidad: Más allá del DSM-IV. Barcelona: Masson.

Millon, T., Davis, R., y Millon, C. (1997). Millon Clinical Multiaxial Inventory-III (MCMI-III) Manual (2nd ed.). Minneapolis: National Computer Systems.

Nunnally, J. (1978). Psychometric Theory (2nd ed.). New York: McGraw-Hill.

Paulhus, D. y Vazire, S. (2007). The selfreport method. En R. Robins, R. Fraley, y R. Krueger (Eds.), Handbook of
Research Methods in Personality Psychology (pp. 224-239). New York: Guilford

Piquero, A., Farrington, D., y Blumstein, A. (2007). Key Issues in Criminal Career Research. New York: Cambridge University Press.

Rossi, G., Van der Ark, L., y Sloore, H. (2007). Factor Analysis of the DutchLanguage Version of the MCMI-III. Journal of Personality Assessment, 88(2), 144-157.

Saulsman, L. y Page, A. (2004). The fivefactor model and personality disorder empirical literature: A meta-analytic review. Clinical Psychology Review, 23, 1055-1085.

Stoner, J. (2008). Using the MCMI in correctional settings. En T. Millon y $\mathrm{C}$. Bloom (Eds.), The Millon Inventories (2nd ed., pp. 196 - 243). New York: Guilford

Strack, S. y Millon, T. (2007). Contributions to the dimensional assessment of personality Disorders using Millon's Model and the Millon Clinical Multiaxial Inventory (MCMI-III). Journal of Personality Assessment, 89(1), 56-69.

Vinet, E., Fuentes, M., Melo, G., Muñoz, R., y Saiz, J. (1998). Necesidades y rasgos: Una réplica transcultural del estudio de Piedmont, McCrae y Costa (1992). Revista de Psicología Social y Personalidad, Vol. XIV, N 1, 29-46.

Vinet, E., Saiz, J., Gempp, R., Benavente, P., Lutz, A., y Pezoa, S. (1999). Examen psicométrico del Inventario Clínico Multiaxial de Millon III (MCMIIII) en una muestra de pacientes chilenos. Terapia Psicológica, 17, 155166.

Widiger, T. y Costa, P. (2009). Five-Factor Model personality disorder research. En P. Costa y T. Widiger (Eds.), Personality Disorders and the Fivefactor Model of Personality (2nd ed., pp. 59-87). Washington: American Psychological Association.

Widiger, T., Trull, T., Clarkin, J., Sanderson, C. y Costa, P. (2009). A description of 
the DSM-IV personality disorders with the five-factor model of personality. En P.Costa y T. Widiger (Eds.), Personality Disorders and the Five-factor Model of Personality (2nd ed., pp. 89-99). Washington: American Psychological Association.

Winberg, M. y Vilalta, R. (2009). Evaluación de trastornos de personalidad mediante el Inventario Clínico Multiaxial (MCMIII) en una muestra forense. Psicothema, 21(4), 610-614. 